\title{
Effect of alpha-lipoic acid on asymmetric dimethylarginine and disability in multiple sclerosis patients: A randomized clinical trial
}

\author{
Mohammad Khalili ${ }^{1}$, Madjid Soltani ${ }^{2}$, Shirin Amiri Moghadam³ ${ }^{3}$ Parvin Dehghan ${ }^{4}$, Amirreza Azimi ${ }^{5}$, Omid \\ Abbaszadeh $^{6}$
}

${ }^{1}$ Ph.D. of Nutrition, Assistant Professor, Neuroscience Research Center, Tabriz University of Medical Sciences, Tabriz, Iran

${ }^{2}$ M.D., Royan Stem Cell Technology, Tehran, Iran

${ }^{3}$ M.D., Ph.D. of Nutrition, Assistant Professor, Department of community medicine, Faculty of Medicine, Hormozgan University of Medical Sciences, Bandar Abbas, Iran

${ }^{4} \mathrm{Ph} . \mathrm{D}$. of Nutrition, Assistant Professor, Department of Food Science and Technology, Faculty of Nutrition and food Sciences, Tabriz University of Medical Sciences, Tabriz, Iran

${ }^{5}$ M.D., Neurologist, Assistant Professor, Multiple Sclerosis Research Center, Tehran University of Medical Sciences, Tehran, Iran

${ }^{6}$ M.D., Research Development and Coordination Center, faculty of Medicine, Deputy of Research and Technology, Tabriz University of Medical Sciences, Tabriz, Iran

Type of article: Original

\begin{abstract}
Background: Multiple Sclerosis (MS) is an inflammatory and demyelinating disease of the central nervous system. Oxidative stress plays a major role in the onset and progression of MS. Asymmetric dimethylarginine (ADMA) formation is dependent on oxidative stress status.

Objective: We examined whether alpha-lipoic acid (ALA) as a potent antioxidant could improve the Expanded Disability Status Scale (EDSS) and decrease plasma level of ADMA in multiple sclerosis patients.

Methods: In a randomized, double-blinded clinical trial conducted at Sina Hospital in Tehran, Iran, from September 2009 to July 2011, 24 patients with relapsing-remitting MS were divided into a treatment group receiving ALA (1200mg/day) for 12 weeks and a control group receiving placebo. Then patients' EDSS and Plasma levels of ADMA were measured at baseline and 12 weeks later. Statistical analysis was done by SPSS software version 16 using the K-S test, Chi square, Mann-Whitney U-test and Wilcoxon test.

Results: The plasma levels of ADMA in the intervention group were decreased significantly $(\mathrm{p}=0.04)$. Also, no patient had increased EDSS score in the supplement group, where 2 out of 12 patients in the placebo group experienced so. Comparing the serum level of ADMA between the two groups failed to show any significant change in the supplement group compared with the control group.

Conclusion: Considering that ADMA is produced by oxidative stress in MS patients and leads to increase of inflammation, ALA may have the potential of beneficial effects in them, in part, by decreasing the plasma level of ADMA and stopping progression.

Trial registration: The trial was registered at the Iranian Registry of Clinical Trials (http://www.irct.ir) with the Irct ID: No. IRCT138812222602N2.

Funding: The authors received no financial support for the research, authorship, and/or publication of this article.

Keywords: Lipoic acid, Multiple sclerosis, Inflammation, Asymmetric dimethylarginine
\end{abstract}

\section{Corresponding author:}

Dr. Madjid Soltani, Royan Stem Cell Technology, Tehran, Iran.

Tel: +98.2127635113, Fax: +98.2189781307, Email: madjsoltani@yahoo.com

Received: July 21, 2016, Accepted: January 14, 2017, Published: July 2017

iThenticate screening: October 09, 2016, English editing: May 17, 2017, Quality control: June 15, 2017

(C) 2017 The Authors. This is an open access article under the terms of the Creative Commons Attribution-NonCommercialNoDerivs License, which permits use and distribution in any medium, provided the original work is properly cited, the use is non-commercial and no modifications or adaptations are made. 


\section{Introduction}

Multiple sclerosis (MS), an inflammatory and demyelinating disease, affects the central nervous system (CNS) by demyelization and destruction of nerve tissue (1). Therefore, inflammation and degenerative processes are the main cascades in the pathogenesis of MS (2). Nitric Oxide (NO), with different biological functions are produced from Larginine via NOS activity. Asymmetric dimethylarginine (ADMA) is a major endogenous inhibitor of endothelial NO synthase (eNOS). Recently, a positive association between inflammatory response and ADMA levels had been found in cardiovascular diseases (3). It is proposed that tissue damage in MS is related to the high levels of NO (4, $5)$. In line with this hypothesis, investigations in $\operatorname{MS}(6,7)$ and experimental allergic encephalomyelitis $(\mathrm{EAE})(8,9)$ have shown that increase in proinflammatory cytokines TNF- $\alpha$ and INF-g is related with consequent progress in iNOS activity and NO generation. Reactive oxygen species (ROS), direct consequence of neuroinflammation, play a major role in inception and development of MS (10). A new report has indicated that oxidative stress increases the ADMA concentration by enhancing the formation and decreasing the degradation of ADMA (11). Alpha-lipoic acid (ALA), a naturally occurring short chain fatty acid with Sulfhydryl groups, has a potent anti-oxidant activity (12). The beneficial effects of lipoic acid on MS patients have been reported in several investigations (13-15). In addition, consumption of ALA led to a drop in plasma ADMA levels in diabetic end-stage renal disease (16). Yet, experimental studies have reported inconsistent roles of NO in the development of neurological immune-pathogen diseases such as multiple sclerosis (MS) $(17,18)$, leaving open the role of NO in this field. In the present study, we assessed whether ALA could improve the Expanded Disability Status Scale (EDSS) and decrease the plasma level of ADMA in multiple sclerosis patients.

\section{Material and Methods}

\subsection{Trial design and participants}

This study was a randomized clinical trial that was conducted from September 2009 to July 2011. This study is a pilot study and, to the best of our knowledge, is the first assessing effect of ALA on ADMA in MS patients. The sample size was assumed to be twenty subjects in each group. The MS patients $(n=40)$ with relapsing-remitting course with disease duration more than one year were recruited from a multiple sclerosis clinic at Sina Hospital in Tehran. Diagnosis of MS was done according to McDonalds criteria (19).

\subsection{Selection criteria}

Excluding criteria were ongoing clinical relapse, pregnancy and lactation, the use of corticosteroids, occurrence of relapse attack during the study, and other major medical disorders. Regular consumption of antioxidants or vitamin supplements was also considered as exclusion criteria.

\subsection{Interventions}

The current study was carried out as a randomized double-blinded clinical trial. During the three months of study, from thirty-one patients allocated to interventional groups (ALA group; $n=15$ and placebo group; $n=16$ ) two patients in the lipoic acid group and one in the placebo group were exposed with relapse and excluded from the study. In addition, a patient in the placebo group not attending the complete study, was excluded. Finally, we finished the study with thirteen patients in the ALA and fourteen patients in the placebo group. At endpoint of study, we lost two blood samples in the placebo group and one blood sample in the supplement group. Therefore, results are presented with twelve participants in each group (Figure 1). While the placebo and ALA capsules were of the same shape and color, participants in ALA and placebo group took $1200 \mathrm{mg}$ of ALA and placebo as two capsules respectively. The EDSS with scores from 0 and 10 units in half units was used to measure the intensity of MS symptoms and to assess the disease status and treatment. On start point and after three months, all participants underwent EDSS evaluation by the same neurologist. Weight was recorded using a Seca Electronic Weighing Scale (Seca, Hamburg, Germany) to the nearest $100 \mathrm{gr}$ wearing light clothing. Height was measured without shoes, using a non-stretchable tape with accuracy of $0.5 \mathrm{~cm}$. Body mass index (BMI) was determined by dividing the weight $(\mathrm{kg})$ by the square of height $\left(\mathrm{m}^{2}\right)$. After overnight fasting, blood samples $(8 \mathrm{ml})$ were obtained at baseline and at week twelve with subjects seated, in accordance with the standard protocol. Serum ADMA was detected by HPLC methodology (20) on HPLC apparatus (Agilent) with our condition modified fluorometric detection. 


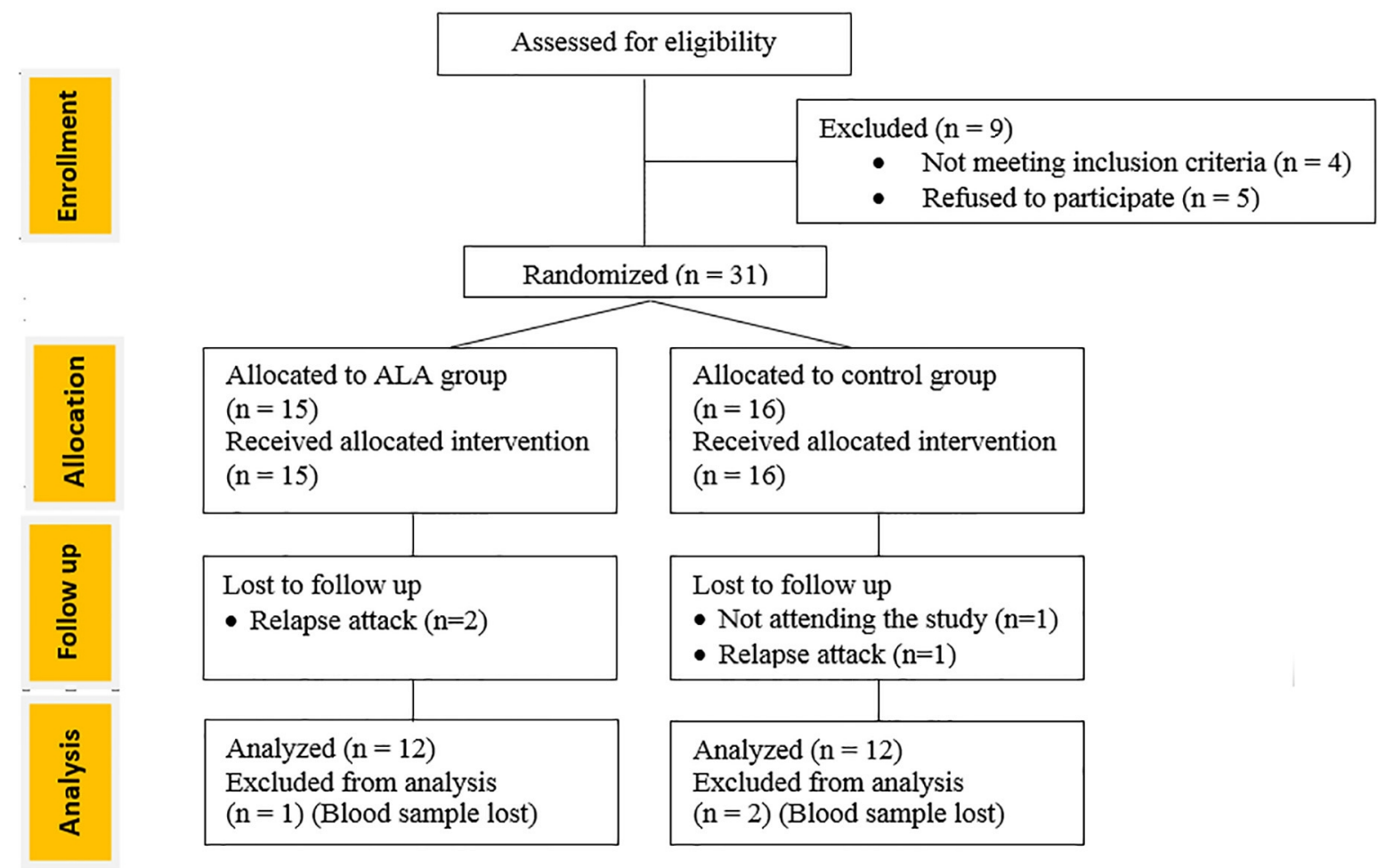

Figure 1. Subjects' disposition

\subsection{Outcomes}

The primary outcomes of our analyses were the mean variation of ADMA and EDSS score during study in the two groups. The secondary outcome was percent of patients exposed with rising of EDSS score in each group.

\subsection{Randomization and blinding}

In this clinical trial, using block randomization, participants were assigned randomly to receive either lipoic acid or placebo. We used computer generated randomization sequence through SPSS16 software (SPSS, Inc., Chicago, IL, USA) by one of the researchers who did not have a role in the treatment of the participants. A block size of four was provided for randomized sequence generation. Also, using numbered envelopes which contained the name of the intervention groups, the allocation hiding was done by the researcher who was responsible for the randomization.

\subsection{Statistical methods}

The mean and standard deviation were calculated using SPSS 16 software (SPSS, Inc., Chicago, IL, USA). The normality of the variables' distribution was examined using the K-S test. Chi square, Mann-Whitney U-test and Wilcoxon tests were used for variables analyzing. The significance level was established at $p$-value $=0.05$.

\subsection{Research ethics}

The study was approved by the Ethics Committee of Tehran University of Medical Sciences. The authors confirm that all ongoing and related trials for this intervention are registered. Prior to the study, all the participants were informed by the researcher about the objective and nature of the study, and provided written consent in Persian language. We were committed to keeping all the participants' information confidential.

\section{Results}

While, the supplement group had higher mean of EDSS than the control group, comparing baseline values of all parameters failed to show any significant difference between the two groups (Table 1). The mean plasma level of ADMA in MS patients was $0.37 \mu \mathrm{mol} / \mathrm{L}$, and baseline level of ADMA difference between supplement and control group was not so significant. In the treatment group, however, intake of ALA for 12 weeks significantly reduced the plasma level of ADMA, from a mean of 0.37 to $0.31 \mu \mathrm{mol} / \mathrm{L}(\mathrm{p}=0.04)$. In the control participants, the plasma level of ADMA showed slight and non-significant increase during the study. Finally, comparing change of ADMA level 
between the two groups did not show statistically significant variation in the ALA group compared with the control group (Table 2). During ALA treatment, EDSS scores in treated patients were slightly and not significant decreasing (at the beginning of the treatment EDSS score was 2.2 \pm 1.6 , after 12 weeks $1.83 \pm 1.5$ ). Instead, EDSS scores did not change significantly in the control group (Table 3 ). In addition, we compared disability progression manner to the number of patients with increased EDSS during intervention. Our findings indicated that in the ALA group, no patient had experience of increased EDSS, but in the placebo group 16.5\% of patients were exposed with raised EDSS after 12 weeks. The correlation coefficients between base line level of ADMA and EDSS as well as the correlation of their changes during the study were not significant (data were not presented).

Table 1. Demographic characteristics at baseline (Mean \pm SD).

\begin{tabular}{|l|l|l|l|}
\hline Variable & Supplement group $(\mathrm{n}=12)$ & Placebo group $(\mathrm{n}=12)$ & $\mathrm{p}$-value \\
\hline Age $($ year $)$ & $32.3 \pm 6.2$ & $32.2 \pm 10.5$ & 0.78 \\
\hline Sex $(\mathrm{F} / \mathrm{M}) \mathrm{n}$ & $7 / 5$ & $11 / 1$ & 0.07 \\
\hline Duration of disease (month) & $53.83 \pm 53.82$ & $69.00 \pm 64.34$ & 0.67 \\
\hline EDSS & $2.20 \pm 1.60$ & $1.54 \pm 1.32$ & 0.31 \\
\hline BMI $\left(\mathrm{kg} / \mathrm{m}^{2}\right)$ & $24.1 \pm 2.9$ & $23.9 \pm 2.3$ & 0.73 \\
\hline
\end{tabular}

Table 2. Baseline and end point level of ADMA and change after placebo or supplement application in patients (Mean \pm SD).

\begin{tabular}{|l|l|l|l|}
\hline Variable & Supplement group $(\mathrm{n}=12)$ & Control group $(\mathrm{n}=12)$ & $\mathrm{p}$-value \\
\hline Base line ADMA $(\mu \mathrm{mol} / \mathrm{L})$ & $0.37 \pm 0.08$ & $0.35 \pm 0.10$ & 0.41 \\
\hline End point ADMA $(\mu \mathrm{mol} / \mathrm{L})$ & $0.31 \pm 0.06$ & $0.37 \pm 0.14$ & 0.19 \\
\hline Difference $(\mu \mathrm{mol} / \mathrm{L})$ & $-0.06 \pm 0.09$ & $0.02 \pm 0.19$ & 0.29 \\
\hline p-value & 0.04 & 0.96 & \\
\hline
\end{tabular}

Table 3. Baseline and end point level of EDSS and change after placebo or supplement application in patients (Mean $\pm \mathrm{SD})$.

\begin{tabular}{|l|l|l|l|}
\hline Variable & Supplement group $(\mathrm{n}=12)$ & Control group $(\mathrm{n}=12)$ & $\mathrm{p}$-value \\
\hline Base line EDSS & $2.20 \pm 1.60$ & $1.54 \pm 1.32$ & 0.31 \\
\hline End point EDSS & $1.83 \pm 1.51$ & $1.54 \pm 1.48$ & 0.67 \\
\hline Difference & $-0.37 \pm 0.77$ & $0.01 \pm 0.1$ & 0.34 \\
\hline p-value & 0.10 & 0.95 & \\
\hline
\end{tabular}

\section{Discussion}

This study is the first to assess the effects of ALA on the plasma level of ADMA in multiple sclerosis patients. In this study, we found that supplementation of ALA (1200mg/day) for 12 weeks, leads to a significant reduction in the plasma level of ADMA in MS patients. It is clear that MS is accompanied by inflammatory process as pathogenesis mechanism. This condition is mediated by activated specific and nonspecific immune cells infiltrating CNS tissue. It has been observed that NO generation is due to induction of inducible nitric oxide synthase (iNOS) in macrophages, increasing NO level is a critical component in the onset and development of MS $(7,21)$. This mechanism is a reason for high levels of NO and iNOS in CNS of both MS (18) and EAE (6). For example, the high levels of NO metabolites in the blood and CSF of MS patients have been observed in independent manner to neurological signs (22). It is believed that reactive nitrogen species, with cytotoxic effects on nerve and glial cells, provoke myelin and oligodendrocytes damage (22). ADMA, a natural amino acid, exits in plasma. It is known that the increase of ADMA, results in a suppression of the NO production by inhibition of NOS activity. Stojanovic and collogues reported that plasma concentration of ADMA in healthy people and MS patients is less than $1 \mu \mathrm{mol} / 1$ (23). Similarly, in our study the ADMA level in MS patients is found in less than $1 \mu \mathrm{mol} / \mathrm{l}$. In another investigation, in patients suffering from multiple sclerosis, CSF ADMA levels were elevated (24). Positive correlation of ADMA levels with intensity of clinical severity was reported in inflammatory bowel disease (25). Furthermore, during interferon- $\beta 1 b$ treatment in multiple sclerosis patients, ADMA levels were significantly increased compared to the baseline levels which were accompanied by decrease in EDSS level in treated patients (23). Regarding our results, treatment with ALA led to a decrease in ADMA levels as well as a halt in disability progression. These facts are in correlation with literature data from an experimental model of MS, reporting that ALA slows down the ICAM-1 and VCAM-1 and suppresses the infiltration of T cells through the blood brain barrier (26-28). Mentioned evidence found that ROS are important factors mediating injury in EAE and that generation of ROS can be decreased by 
ALA. Also, ALA decreased the migration of monocytes through the blood brain barrier, which was correlated with the clinical improvement in EAE. However, there is no clear evidence evaluating direct correlation of ADMA level with clinical severity of MS. This study showed that ALA decreases circulating ADMA concentrations in patients with multiple sclerosis. Similarly, Chang and colleagues found that consumption of LA $(600 \mathrm{mg} /$ day $)$ decreased ADMA levels in a treated group of diabetic end-stage renal disease (16). ALA, as a potent antioxidant and antiinflammation supplement, has been used in treatment of MS and suppressed proinflammatory cytokines levels such as TNF- $\alpha$ and INF-g in EAE models and MS patients $(28,29)$. In agreement with our results, researchers indicate that ALA decreases ADMA level in diabetes mellitus (30). ADMA is excreted via the kidneys and mainly metabolized by the enzyme dimethylarginine dimethylamino hydrolase (DDAH) (31), which is sensitive to oxidative stress (32). It is proposed that ALA via antioxidant properties could influence DDAH. Hence, accumulation of ADMA in nerve cells due to produced proinflammatory cytokines, such as TNF- $\alpha$ (33) as well as suppressing effect of ALA on TNF- $\alpha$ level in MS patients $(15,29)$ may be explanations for our results.

\section{Limitation}

The limitation of this study is that it does not show the clear mechanism by which ALA decreases the plasma level of ADMA. In addition, this study did not consider the potential for NO generation by the ADMA or give information on the activity of the DDAH enzyme that degrades ADMA.

\section{Conclusions}

Regarding our findings, ALA supplementation decreased ADMA levels and led to observe no patient with increased disability score during the study. But decrease of disability score in the ALA group was not significant. Considering that ADMA is produced by inflammation, however, ALA could have the potential to have beneficial effects on MS patients. We think that further studies with large samples and extended duration are needed to prove the effectiveness of ALA on the progression of multiple sclerosis.

\section{Acknowledgments:}

This paper was extracted from a nutrition PhD thesis at Tehran University of Medical Sciences in Tehran, Iran. The authors appreciate the assistance and cooperation provided by the staff members in the Sina and Emam Khomeyni Hospitals. Also, we appreciate the assistance provided by Mr. Meysam Sanoobar (MSc in Nutrition) and Dr. Ghazaleh Skandari (MD). Authors thank all patients who participated in study.

\section{Conflict of Interest:}

There is no conflict of interest to be declared.

\section{Authors' contributions:}

All authors contributed to this project and article equally. All authors read and approved the final manuscript.

\section{References:}

1) Babaloo Z, Babaie F, Farhoodi M, Aliparasti MR, Baradaran B, Almasi S, et al. Interleukin-17A and Interleukin-17F mRNA Expressions in Peripheral Blood Mononuclear Cells of Patients with Multiple Sclerosis. Iran J Immunol. 2010; 7(4): 202-9. doi: IJIv7i4A1. PMID: 21189442.

2) Lassmann $\mathrm{H}$, Brück $W$, Lucchinetti C. Heterogeneity of multiple sclerosis pathogenesis: implications for diagnosis and therapy. Trends Mol Med. 2001, 7(3): 115-21. doi: 10.1016/S1471-4914(00)01909-2. PMID: 11286782 .

3) Sandoo A, Dimitroulas T, Hodson J, Smith JP, Douglas KM, Kitas GD. Cumulative inflammation associates with asymmetric dimethylarginine in rheumatoid arthritis: a 6 year follow-up study. Rheumatology (Oxford). 2015; 54(7): 1145-52. doi: 10.1093/rheumatology/keu349. PMID: 25187642.

4) Ghasemi M, Fatemi A. Pathologic role of glial nitric oxide in adult and pediatric neuroinflammatory diseases. Neurosci Biobehav Rev. 2014; 45: 168-82. doi: 10.1016/j.neubiorev.2014.06.002. PMID: 24933692.

5) Smith KJ, Kapoor R, Felts PA. Demyelination: the role of reactive oxygen and nitrogen species. Brain Pathol. 1999; 9(1): 69-92. doi: 10.1111/j.1750-3639.1999.tb00212.x. PMID: 9989453.

6) Bö L, Dawson TM, Wesselingh S, Möurk S, Choi S, Kong PA, et al. Induction of nitric oxide synthase in demyelinating regions of multiple sclerosis brains. Ann Neurol. 1994; 36(5): 778-86. doi: 10.1002/ana.410360515. PMID: 7526776. 
7) Bagasra O, Michaels FH, Zheng YM, Bobroski LE, Spitsin SV, Fu ZF, et al. Activation of the inducible form of nitric oxide synthase in the brains of patients with multiple sclerosis. Proc Natl Acad Sci U S A. 1995; 92(26): 12041-5. doi: 10.1073/pnas.92.26.12041. PMID: 8618840, PMCID: PMC40292.

8) Endoh M, Maiese K, Wagner J. Expression of the inducible form of nitric oxide synthase by reactive astrocytes after transient global ischemia. Brain Res. 1994; 651(1-2): 92-100. doi: 10.1016/00068993(94)90683-1. PMID: 7522935.

9) Lin RF, Lin TS, Tilton RG, Cross AH. Nitric oxide localized to spinal cords of mice with experimental allergic encephalomyelitis: an electron paramagnetic resonance study. J Exp Med. 1993; 178(2): 643-8. doi: 10.1084/jem.178.2.643. PMID: 8393479, PMCID: PMC2191106.

10) Gilgun-Sherki Y, Melamed E, Offen D. The role of oxidative stress in the pathogenesis of multiple sclerosis: the need for effective antioxidant therapy. J Neurol. 2004; 251(3): 261-8. doi: 10.1007/s00415004-0348-9. PMID: 15015004.

11) Luo Y, Yue W, Quan X, Wang Y, Zhao B, Lu Z. Asymmetric dimethylarginine exacerbates A $\beta$-induced toxicity and oxidative stress in human cell and Caenorhabditis elegans models of Alzheimer disease. Free Radic Biol Med. 2015; 79: 117-26. doi: 10.1016/j.freeradbiomed.2014.12.002. PMID: 25499850.

12) Moini H, Packer L, Saris NE. Antioxidant and prooxidant activities of $\alpha$-lipoic acid and dihydrolipoic acid. Toxicol Appl Pharmacol. 2002; 182(1): 84-90. doi:10.1006/taap.2002.9437. PMID: 12127266.

13) Khalili M, Eghtesadi S, Mirshafiey A, Eskandari G, Sanoobar M, Sahraian MA, et al. Effect of lipoic acid consumption on oxidative stress among multiple sclerosis patients: A randomized controlled clinical trial. Nutr Neurosci. 2014; 17(1): 16-20. doi: 10.1179/1476830513Y.0000000060. PMID: 23485514.

14) Yadav V, Marracci G, Lovera J, Woodward W, Bogardus K, Marquardt W, et al. Lipoic acid in multiple sclerosis: a pilot study. Mult Scler. 2005; 11(2): 159-65. doi: 10.1191/1352458505ms1143oa. PMID: 15794388.

15) Salinthone S, Yadav V, Bourdette DN, Carr DW. Lipoic acid: a novel therapeutic approach for multiple sclerosis and other chronic inflammatory diseases of the CNS. Endocr Metab Immune Disord Drug Targets. 2008; 8(2): 132-42. doi: 10.2174/187153008784534303. PMID: 18537699.

16) Chang JW, Lee EK, Kim TH, Min WK, Chun S, Lee KU, et al. Effects of $\alpha$-lipoic acid on the plasma levels of asymmetric dimethylarginine in diabetic end-stage renal disease patients on hemodialysis: a pilot study. Am J Nephrol. 2007; 27(1): 70-4. doi: 10.1159/000099035. PMID: 17259696.

17) Mitrovic B, Ignarro LJ, Vinters HV, Akers MA, Schmid I, Uittenbogaart C, et al. Nitric oxide induces necrotic but not apoptotic cell death in oligodendrocytes. Neuroscience. 1995; 65(2): 531-9. doi: 10.1016/0306-4522(94)00491-M. PMID: 7777166.

18) Smith KJ, Lassmann H. The role of nitric oxide in multiple sclerosis. Lancet Neurol. 2002; 1(4): $232-41$. doi: 10.1016/S1474-4422(02)00102-3. PMID: 12849456.

19) Polman CH, Reingold SC, Banwell B, Clanet M, Cohen JA, Filippi M, et al. Diagnostic criteria for multiple sclerosis: 2010 revisions to the McDonald criteria. Ann Neurol. 2011; 69(2): 292-302. doi: 10.1002/ana.22366. PMID: 21387374, PMCID: PMC3084507.

20) Pi J, Kumagai Y, Sun G, Shimojo N. Improved method for simultaneous determination of L-arginine and its mono-and dimethylated metabolites in biological samples by high-performance liquid chromatography. J Chromatogr B Biomed Sci Appl. 2000; 742(1): 199-203. doi: 10.1016/S0378-4347(00)00145-6. PMID: 10892599.

21) Koprowski H, Zheng YM, Heber-Katz E, Fraser N, Rorke L, Fu ZF, et al. In vivo expression of inducible nitric oxide synthase in experimentally induced neurologic diseases. Proc Natl Acad Sci U S A. 1993; 90(7): 3024-7. doi: 10.1073/pnas.90.7.3024. PMID: 7681993, PMCID: PMC46229.

22) Johnson AW, Land JM, Thompson EJ, Bolaños JP, Clark JB, Heales SJ. Evidence for increased nitric oxide production in multiple sclerosis. J Neurol Neurosurg Psychiatry. 1995; 58(1): 107. doi: 10.1136/jnnp.58.1.107. PMID: 7823050, PMCID: PMC1073282.

23) Stojanovic I, Vojinovic S, Ljubisavljevic S, Pavlovic R, Basic J, Pavlovic D, et al. INF- $\beta 1$ b therapy modulates 1-arginine and nitric oxide metabolism in patients with relapse remittent multiple sclerosis. J Neurol Sci. 2012; 323(1-2): 187-92. doi: 10.1016/j.jns.2012.09.014. PMID: 23026532.

24) Conte C, Floridi E, Galli F, Aisa C, Floridi A. A new method for the liquid chromatography analysis of methylated arginines in biological fluids and tissues by o-phthaldialdehyde postcolumn derivatization. Anal Biochem. 2005; 338(2): 347-9. doi: 10.1016/j.ab.2004.11.018. PMID: 15745758.

25) Owczarek D, Cibor D, Mach T. Asymmetric dimethylarginine (ADMA), symmetric dimethylarginine (SDMA), arginine, and 8 - iso - prostaglandin F2 a (8 - iso - PGF2 a ) level in patients with inflammatory bowel diseases. Inflamm Bowel Dis. 2010; 16(1): 52-7. doi: 10.1002/ibd.20994. PMID: 19575355. 
26) Schreibelt G, Musters RJ, Reijerkerk A, de Groot LR, van der Pol SM, Hendrikx EM, et al. Lipoic acid affects cellular migration into the central nervous system and stabilizes blood-brain barrier integrity. J Immunol. 2006; 177(4): 2630-7. doi: 10.4049/jimmunol.177.4.2630. PMID: 16888025.

27) Marracci GH, McKeon GP, Marquardt WE, Winter RW, Riscoe MK, Bourdette DN. Alpha lipoic acid inhibits human T-cell migration: implications for multiple sclerosis. J Neurosci Res. 2004; 78(3): 362-70. doi: 10.1002/jnr.20255. PMID: 15389837.

28) Chaudhary P, Marracci GH, Bourdette DN. Lipoic acid inhibits expression of ICAM-1 and VCAM-1 by CNS endothelial cells and $\mathrm{T}$ cell migration into the spinal cord in experimental autoimmune encephalomyelitis. J Neuroimmunol. 2006; 175(1-2): 87-96. doi: 10.1016/j.jneuroim.2006.03.007. PMID: 16644024.

29) Khalili M, Azimi A, Izadi V, Eghtesadi S, Mirshafiey A, Sahraian MA, et al. Does Lipoic Acid Consumption Affect the Cytokine Profile in Multiple Sclerosis Patients: A Double-Blind, PlaceboControlled, Randomized Clinical Trial. Neuroimmunomodulation. 2014; 21(6): 291-6. doi: 10.1159/000356145. PMID: 24821457.

30) Mittermayer F, Pleiner J, Francesconi M, Wolzt M. Treatment with $\alpha$-lipoic acid reduces asymmetric dimethylarginine in patients with type 2 diabetes mellitus. Transl Res. 2010; 155(1): 6-9. doi: 10.1016/j.trsl.2009.08.004. PMID: 20004356.

31) Vallance P, Leiper J. Cardiovascular biology of the asymmetric dimethylarginine: dimethylarginine dimethylaminohydrolase pathway. Arterioscler Thromb Vasc Biol. 2004; 24(6): 1023-30. doi: 10.1161/01.ATV.0000128897.54893.26. PMID: 15105281.

32) Lin KY, Ito A, Asagami T, Tsao PS, Adimoolam S, Kimoto M, et al. Impaired nitric oxide synthase pathway in diabetes mellitus role of asymmetric dimethylarginine and dimethylarginine dimethylaminohydrolase. Circulation. 2002; 106(8): 987-92. doi: 10.1161/01.CIR.0000027109.14149.67. PMID: 12186805.

33) Leiper J, Vallance P. Biological significance of endogenous methylarginines that inhibit nitric oxide synthases. Cardiovasc Res. 1999; 43(3): 542-8. doi: 10.1016/S0008-6363(99)00162-5. PMID: 10690326. 